\title{
Field-induced coordinates for the determination of dynamic vibrational nonlinear optical properties
}

\author{
Josep M. Luis and Miquel Duran \\ Institute of Computational Chemistry and Department of Chemistry, University of Girona, \\ Campus de Montilivi, 17071 Girona, Catalonia, Spain \\ Bernard Kirtman \\ Department of Chemistry and Biochemistry, University of California, Santa Barbara, California 93106
}

(Received 27 April 2001; accepted 18 June 2001)

\begin{abstract}
The most important contribution to nonresonant vibrational nonlinear optical properties arises from nuclear relaxation (NR). In previous work a set of static field-induced vibrational coordinates (FICs) has been developed to simplify calculation of the NR contribution within the infinite optical frequency approximation. Although the number of FICs is small and independent of molecular size, these coordinates form a complete set. However, the infinite optical frequency approximation does not take into account the frequency dispersion, which we evaluate for three conjugated organic molecules that span a range of polarity and valence-bond/charge transfer characteristics. Our results show that dispersion can be significant and that, in such cases, frequency-dependent FICs (FD-FICs) are necessary for an adequate treatment. A complete, though still small, set of FD-FICs is presented for this purpose. Computational tests reveal that a reduced subset, together with an harmonic approximation, can be used to achieve high accuracy outside the infrared (IR) region. That subset is complete for the electro-optic and Pockels and Kerr effects though not for other common properties. (C) 2001 American Institute of Physics. [DOI: 10.1063/1.1390525]
\end{abstract}

\section{INTRODUCTION}

It is well-established that vibrational motions often play a major role in nonlinear optical (NLO) properties. ${ }^{1-4}$ For nonresonant processes there exists a strong theoretical edifice whereby that role may be quantified through quantum chemical computations of the relevant molecular hyperpolarizabilities at various levels of approximation. Thus, an appropriate frequency-dependent perturbation treatment ${ }^{5-7}$ has been developed by Bishop and Kirtman (BK). It is based on a clamped nucleus approximation, ${ }^{8}$ which separates electronic from nuclear motions, and is based on a doubly harmonic (mechanical and electrical) zeroth-order model. Compact vibrational (hyper)polarizability formulas, complete through second-order in mechanical plus electrical anharmonicity, have been derived from this treatment. ${ }^{9}$ If we set aside the contribution from the zero-point vibrational average (ZPVA), for the moment, then each term in the perturbation expansion contains products of derivatives of lower-order electronic properties with respect to vibrational coordinates. This provides a basis for classifying the terms into unique "square bracket" types. For example, the vibrational second hyperpolarizability $\gamma^{v}\left(-\omega_{\sigma} ; \omega_{1}, \omega_{2}, \omega_{3}\right)$ contains terms of the $\left[\mu^{2} \alpha\right]$ square bracket type, meaning a product of two factors with derivatives of the electronic dipole moment $\mu^{e}$ and one factor with a derivative of the electronic linear polarizability $\alpha^{e}$ is involved. Here $\omega_{1}, \omega_{2}$, and $\omega_{3}$ are the frequencies of the three applied fields; $\omega_{\sigma}=\omega_{1}+\omega_{2}+\omega_{3}$; and the perturbation terms also depend on these frequencies as well as the vibrational force constants. It turns out that the $\left[\mu^{2} \alpha\right]$ contribution vanishes in zeroth-order as well as in all other even orders. This pattern varies (in a systematic way) for different square bracket types, e.g., $\left[\alpha^{2}\right]$ terms contribute in zerothorder, but vanish in all odd orders; on the other hand, the first nonvanishing $\left[\mu^{4}\right]$ terms are second-order while all subsequent odd orders vanish.

The lowest-order nonvanishing terms of each type, taken in toto, constitute the nuclear relaxation (NR) approximation. This name arises from considering the case where one or more of the applied fields is static and the remaining optical fields (if any) are formally allowed to become infinite. ${ }^{10} \mathrm{In}$ that event the static field(s) will induce a shift in the equilibrium geometry (i.e., a nuclear relaxation) while the nuclei will be totally unresponsive to the optical fields. If there were no NR, then an expansion of the static electronic dipole moment, $\mu^{e}(0)$, as a power series in the static field(s) would give $\alpha^{e}(0 ; 0)$ as the linear term, $\beta^{e}(0 ; 0,0)$ as the quadratic term, and $\gamma^{e}(0 ; 0,0,0)$ as the cubic term. When the nuclear relaxation is taken into account this expansion gives, in addition, the NR contributions $\alpha^{\mathrm{nr}}(0 ; 0), \quad \beta^{\mathrm{nr}}(0 ; 0,0)$, and $\gamma^{\mathrm{nr}}(0 ; 0,0,0) .{ }^{11}$ Similarly, from the corresponding expansion of $\alpha^{e}(0 ; 0)$ we get $\beta^{\mathrm{nr}}(-\omega ; \omega, 0)_{\omega \rightarrow \infty}$ and $\gamma^{\mathrm{nr}}(-\omega ; \omega, 0,0)_{\omega \rightarrow \infty} ;$ and from $\beta^{e}(0 ; 0,0)$ we obtain $\gamma^{\operatorname{nr}}(-2 \omega ; \omega, \omega, 0)_{\omega \rightarrow \infty} .{ }^{12}$ To complete the picture it should be evident that both $\beta^{\text {nr }}(-2 \omega ; \omega, \omega)_{\omega \rightarrow \infty}$ and $\gamma^{\mathrm{nr}}(-3 \omega ; \omega, \omega, \omega)_{\omega \rightarrow \infty}$ are zero. The NR (hyper)polarizabilities found in this fashion turn out to be identical to the corresponding properties calculated as the sum of lowest-order nonvanishing perturbation terms of each square bracket type. ${ }^{10,13}$

Clearly, the static and infinite optical frequency NR properties listed above can be determined by finite field (FF) computations. Included are all but one (see below) of the 
most commonly studied NLO processes. Although care must be taken to avoid molecular rotations with respect to the field, ${ }^{14}$ the FF method has proven to be an attractive way to calculate infinite optical frequency properties - to be further discussed later on - not only for individual molecules, ${ }^{15}$ but also for molecules in solution ${ }^{16}$ and for the linear polarizability of infinite polymers. ${ }^{17}$ It is evident, however, that this procedure cannot be employed for NR properties at arbitrary frequencies. Thus, it cannot be applied to obtain frequency dispersion nor even to obtain $\gamma^{\mathrm{nr}}(-\omega ; \omega,-\omega, \omega)_{\omega \rightarrow \infty}$. The value for the latter quantity found from the perturbation treatment is simply the static zeroth-order $\left[\alpha^{2}\right]$ multiplied by $2 / 3$.

An analogous FF treatment has been developed for the next higher-order (nonvanishing) terms of each type in the BK perturbation expansion. For this purpose the static electronic properties are replaced by their static first-order ZPVA corrections. ${ }^{18}$ In evaluating these corrections one must take into account the fact that the vibrational wave functions are field-dependent. Relaxation (i.e., change due to the field) of the vibrational wave functions is caused only in part by relaxation of the nuclear geometry. For this, and for historical reasons, the terms that replace $\alpha^{\mathrm{nr}}, \beta^{\mathrm{nr}}, \gamma^{\mathrm{nr}}$ are denoted by $\alpha^{\text {c-zpva }}, \beta^{\text {c-zpva }}, \gamma^{\text {c-zpva }}$, where $\mathrm{C}$ refers to the curvature of the field-dependent vibrational potential. The key point is that the C-ZPVA terms constitute the next higher-order (nonvanishing) perturbation contributions of each type to the static and infinite optical frequency vibrational (hyper)polarizabilities. Likewise, using the second-order ZPVA leads to the next succeeding higher-order (nonvanishing) perturbation terms of each type, and so forth, until the entire perturbation series is accounted for. ${ }^{19}$

As already noted the FF approach does not take into account frequency dispersion. There have been some studies of NR (i.e., $\alpha^{\mathrm{nr}}, \beta^{\mathrm{nr}}, \gamma^{\mathrm{nr}}$ ) in small molecules ${ }^{20,21}$ showing that dispersion is relatively small whenever this contribution is important. Similar investigations have not been conducted, however, for conjugated organic molecules of the sort that are of interest as NLO materials. That is one purpose of this paper. The other is to present a convenient analytic method for computing the frequency-dependence using so-called field-induced coordinates (FICs). We note here that an alternative scheme for generalizing the FF procedure to accomplish the same purpose has previously been proposed ${ }^{22}$ but it is more difficult to implement and does not apply to all NLO properties.

It turns out that the $3 N-6$ normal coordinates employed in the perturbation treatment of vibrational NLO properties are more than needed to determine the NR terms. Within the infinite optical frequency approximation it has been demonstrated ${ }^{14,23}$ analytically (and numerically) that a small set of static FICs, which remain fixed in number regardless of molecular size, is able to duplicate exactly the result obtained with all $3 N-6$ normal modes. ${ }^{24}$ These coordinates can be used directly to simplify and evaluate the perturbation theory formulas for the static and infinite optical frequency NR (hyper)polarizabilities. Other static FICs can be used to advantage to determine the corresponding ZPVA and C-ZPVA hyperpolarizabilities ${ }^{25}$ while still others are readily developed for efficient calculation of vibrational effects in a wide variety of electrical, magnetic and spectroscopic properties. ${ }^{26}$

In the next section a small set of dynamic or frequencydependent FICs (i.e., FD-FICs) is defined which proved to be sufficient to give the exact frequency-dependent NR (hyper)polarizability for any process. General formulas for the (hyper)polarizabilities in terms of the FD-FICs are presented. For the most common NLO properties the static FICs used previously will occur as a subset of the dynamic FICs. However, there are other interesting cases where that is not so. An example is sum-frequency generation (SFG) involving the second-order NLO response (i.e., first hyperpolarizability) as applied either to chiral molecules ${ }^{27,28}$ or to surface vibrational spectroscopy with one fixed frequency in the UV domain and one tunable infrared frequency. ${ }^{29}$

Section III contains test calculations of the most common NLO properties for three $\pi$-conjugated molecules spanning a range of polarity and valence bond-charge transfer characteristics. Our frequency-dependent results allow us to make an initial assessment of the conditions likely to yield significant errors when the infinite optical frequency approximation (no dispersion) is applied. Frequency dispersion is introduced approximately even if only static FICs are employed in the frequency-dependent BK perturbation theory expressions. Numerical tests reveal that this procedure is not satisfactory and, therefore, that FD-FICs are required. In addition to the complete set of FD-FICs we have found a reduced set that leads to only a small loss in accuracy outside the IR region and, further, that neglecting anharmonicity contributions is a good approximation. This reduced set is exact for the electro-optic Pockels and Kerr effects though not for other NLO properties. Finally, in Sec. IV we review our conclusions and present some plans for future work.

\section{FREQUENCY DEPENDENT FIELD INDUCED COORDINATES}

Under the influence of a uniform static electric field, $\mathbf{F}$, the equilibrium geometry of a molecule will relax to a new field-dependent equilibrium position. We call this change in geometry nuclear relaxation (NR). The value of the $i$ th fieldfree normal coordinate induced by NR may be written as a power series in the field,

$$
\begin{aligned}
Q_{i}^{F}\left(F_{x}, F_{y}, F_{z}\right)= & \sum_{\alpha}^{x, y, z} \frac{\partial Q_{i}^{F}}{\partial F_{\alpha}} F_{\alpha}+\frac{1}{2} \sum_{\alpha, \beta}^{x, y, z} \frac{\partial^{2} Q_{i}^{F}}{\partial F_{\alpha} \partial F_{\beta}} F_{\alpha} F_{\beta} \\
& +\cdots .
\end{aligned}
$$

Expansion of the potential energy as a double power series in terms of the normal coordinates and $\mathbf{F}$, followed by energy minimization, ${ }^{1,11}$ leads to expressions for the derivatives of $Q_{i}^{F}$ that appear in Eq. (1),

$$
\frac{\partial Q_{i}^{F}}{\partial F_{\alpha}}=-q_{1}^{i, \alpha},
$$




$$
\begin{aligned}
\frac{1}{2} \frac{\partial^{2} Q_{i}^{F}}{\partial F_{\alpha} \partial F_{\beta}}= & -q_{2}^{i, \alpha \beta}+\sum_{j=1}^{3 N-6} \frac{a_{21}^{i j, \alpha}}{a_{20}^{i i}} q_{1}^{i, \beta} \\
& -\sum_{j, k=1}^{3 N-6} \frac{3 a_{30}^{i j k}}{2 a_{20}^{i i}} q_{1}^{j, \alpha} q_{1}^{k, \beta},
\end{aligned}
$$

where

$$
\begin{aligned}
& a_{n m}^{i, j \ldots, \alpha, \beta, \ldots} \\
& \quad=\frac{1}{n ! m !}\left(\frac{\partial^{(n+m)} V\left(Q_{1}, \ldots, Q_{3 N-6}, F_{x}, F_{y}, F_{z}\right)}{\partial Q_{i} \partial Q_{j} \cdots \partial F_{\alpha} \partial F_{\beta} \cdots}\right)_{Q=0, F=0},
\end{aligned}
$$

$q_{1}^{i, \alpha}=\frac{a_{11}^{i, \alpha}}{2 a_{20}^{i i}}$,

$q_{2}^{i, \alpha \beta}=\frac{a_{12}^{i, \alpha \beta}}{2 a_{20}^{i i}}$,

and the sums over $3 N-6$ normal coordinates reduce to $3 N$ -5 for linear molecules. The coefficient $a_{10}^{i}$ is zero, $a_{20}^{i i}$ is equal to $\frac{1}{2} \omega_{i}^{2}$, with $\omega_{i}$ being the harmonic vibrational frequency, and the $a_{n m}$ with $n=1$ and $m>0$ are (harmonic) electrical property first derivatives. For $n>2$ and $m=0$ the $a_{n m}$ are mechanical anharmonicity parameters; for $n>1$ and $m>0$ these coefficients characterize the electrical anharmonicity.

From Eqs. (2) and (3) the static linear and quadratic FICs are defined $b y^{23}$

$$
\begin{aligned}
\chi_{1}^{\alpha}= & \sum_{i=1}^{3 N-6} \frac{\partial Q_{i}^{F}}{\partial F_{\alpha}} Q_{i}=-\sum_{i=1}^{3 N-6} q_{1}^{i, \alpha} Q_{i}, \\
\chi_{2}^{\alpha \beta}= & \frac{1}{2} \sum_{i=1}^{3 N-6} \frac{\partial^{2} Q_{i}^{F}}{\partial F_{\alpha} \partial F_{\beta}} Q_{i} \\
= & \sum_{i=1}^{3 N-6}\left[-q_{2}^{i, \alpha \beta}+\sum_{j=1}^{3 N-6} \frac{a_{21}^{i j, \alpha}}{a_{20}^{i i}} q_{1}^{j, \beta}\right. \\
& \left.-\sum_{j, k=1}^{3 N-6} \frac{3 a_{30}^{i j k}}{2 a_{20}^{i i}} q_{1}^{j, \alpha} q_{1}^{k, \beta}\right] Q_{i} .
\end{aligned}
$$

Whereas the first-order FICs $\left(\chi_{1}^{\alpha}\right)$ depend only on harmonic $a_{n m}$, the second-order FICs $\left(\chi_{2}^{\alpha \beta}\right)$ depend also on the anharmonicity parameters $a_{21}$ and $a_{30}$. The latter are far more difficult to compute than the corresponding harmonic parameters $a_{11}$ and $a_{20}$ since they involve higher-order differentiation and occur in greater number. Removing the anharmonic terms from Eq. (8) one can define the harmonic second-order static FIC,

$$
\chi_{2, \mathrm{har}}^{\alpha \beta}=\frac{1}{2} \sum_{i=1}^{3 N-6}\left(\frac{\partial^{2} Q_{i}^{F}}{\partial F_{\alpha} \partial F_{\beta}}\right) Q_{\mathrm{har}} Q_{i}=-\sum_{i=1}^{3 N-6} q_{2}^{i, \alpha \beta} Q_{i} .
$$

As mentioned in the Introduction the static FICs are advantageous over normal modes because they reduce the number of coordinates needed to calculate the NR contribution to electric, magnetic and spectroscopic properties ${ }^{19}$ in the infinite optical frequency approximation ${ }^{12}$ (and in the static case). For instance, the (approximate) NR contribution to the diagonal elements of the IDRI tensor contains terms involving all $3 N-6$ normal coordinates, ${ }^{10,12}$ but when static FICs are used instead only one vibrational coordinate $\left(\chi_{2, \text { har }}^{\alpha \alpha}\right)$ for each Cartesian direction is needed, ${ }^{23,24}$ i.e.,

$$
\begin{aligned}
\gamma_{\alpha \alpha \alpha \alpha}^{\mathrm{nr}}(-\omega ; \omega,-\omega, \omega)_{\omega \rightarrow \infty} & =2 \sum_{i=1}^{3 N-6} \frac{\partial \alpha_{\alpha \alpha}}{\partial Q_{i}}\left(\frac{\partial^{2} Q_{i}^{F}}{\partial F_{\alpha}^{2}}\right)_{\text {har }} \\
& =2 \frac{\partial \alpha_{\alpha \alpha}}{\partial \chi_{2, \text { har }}^{\alpha}}\left(\frac{\partial^{2} \chi_{2, \text { har }}^{F}}{\partial F_{\alpha}^{2}}\right)_{\text {har }} .
\end{aligned}
$$

Note that Eq. (10) is exact. In addition, the static FICs given by Eqs. (7)-(9) permit a similar simplification for all NLO processes that involve at least one static field such as $\gamma^{\mathrm{nr}}(-\omega ; \omega, 0,0)_{\omega \rightarrow \infty}$.

The static FICs yield exact results only in the static case and in the infinite optical frequency approximation. It is desirable to have frequency-dependent FICs (FD-FICs) that can be used when this approximation is not sufficient to determine $\quad \alpha^{\mathrm{nr}}\left(-\omega_{\sigma} ; \omega_{1}\right), \quad \beta^{\mathrm{nr}}\left(-\omega_{\sigma} ; \omega_{1}, \omega_{2}\right), \quad$ and $\gamma^{\mathrm{nr}}\left(-\omega_{\sigma} ; \omega_{1}, \omega_{2}, \omega_{3}\right)$ for arbitrary frequencies. To that end we start with the expressions for the exact frequencydependent NR contribution to the dynamic (hyper)polarizabilities as derived by BK. ${ }^{6}$ Thus, for the linear polarizability,

$$
\begin{aligned}
\alpha_{\alpha \beta}^{\mathrm{nr}}(-\omega ; \omega) & =\frac{1}{2} \sum P_{\alpha \beta} \sum_{i=1}^{3 N-6} \frac{a_{11}^{i, \alpha} a_{11}^{i, \beta}}{2 a_{20}^{i i}-\omega^{2}} \\
& =\frac{1}{2} \sum P_{\alpha \beta} \sum_{i=1}^{3 N-6} a_{11}^{i, \alpha} q_{1,|\omega|}^{i, \beta},
\end{aligned}
$$

where

$$
q_{1,|\omega|}^{i, \beta}=\frac{a_{11}^{i, \beta}}{2 a_{20}^{i i}-\omega^{2}},
$$

and $\sum P_{\alpha \beta}$ indicates a sum over both permutations of the indices $\alpha$ and $\beta$. Since $q_{1,|\omega|}^{i, \alpha}$ reduces to $q_{1}^{i, \alpha}$ when $\omega=0$ this suggests that Eq. (7) for the first-order static FIC can be generalized to the frequency-dependent form,

$$
\chi_{1,|\omega|}^{\alpha}=-\sum_{i=1}^{3 N-6} q_{1,|\omega|}^{i, \alpha} Q_{i} .
$$

This defines the first-order FD-FIC. Similarly, one can replace $q_{1}^{i, \alpha}$ in Eq. (2) with $q_{1,|\omega|}^{i, \alpha}$ and, simultaneously, $F_{\alpha}$ with $F_{\alpha}^{|\omega|}$ to obtain the frequency-dependent linear nuclear relaxation (per unit field),

$$
\frac{\partial Q_{i}^{F}}{\partial F_{\alpha}^{|\omega|}}=-q_{1,|\omega|}^{i, \alpha} .
$$

It is easy to demonstrate that $\alpha_{\alpha \beta}^{\mathrm{nr}}(\omega ; \omega)$ can be written exactly in terms of $\chi_{1,|\omega|}^{\alpha}$ and $\chi_{1,|\omega|}^{\beta}$ without invoking any other coordinates. The first step is to construct two separate sets of vibrational coordinates, which are linear combinations of the field-free normal coordinates, such that $\phi_{1}^{\alpha}$ 
$=\chi_{1, \omega \mid}^{\alpha} \quad\left(\phi_{1}^{\beta}=\chi_{1,|\omega|}^{\beta}\right) \quad$ and $\quad\left\{\phi_{2}^{\alpha}, \phi_{3}^{\alpha}, \cdots, \phi_{3 N-6}^{\alpha}\right\}$ $\left(\left\{\phi_{2}^{\beta}, \phi_{3}^{\beta}, \cdots, \phi_{3 N-6}^{\beta}\right\}\right)$ is the orthogonal complement of $\phi_{1}^{\alpha}\left(\phi_{1}^{\beta}\right)$. Hence,

$$
\phi_{i}^{\alpha}=\sum_{j=1}^{3 N-6} M_{i j}^{\alpha,|\omega|} Q_{j}
$$

where $\mathbf{M}^{\alpha,|\omega|}$ is an orthogonal matrix and $M_{1 j}^{\alpha,|\omega|}=-q_{1,|\omega|}^{j, \alpha}$. There is, of course, an exactly analogous transformation matrix $\mathbf{M}^{\beta,|\omega|}$ that determines the coordinate set $\left\{\phi_{1}^{\beta}\right\}$. Note that the same transformation as in Eq. (15) connects the linear relaxation of the coordinates $\phi_{i}$ with the linear relaxation of the normal coordinates, i.e.,

$$
\phi_{i}^{F}=\sum_{j=1}^{3 N-6} M_{i j}^{\alpha,|\omega|} Q_{j}^{F}
$$

Thus, by applying the chain rule to determine $\partial \phi_{1}^{F} / \partial F_{\alpha}^{|\omega|}$,

$$
\sum_{i}^{3 N-6} M_{1 i}^{\alpha,|\omega|} M_{1 i}^{\alpha,|\omega|}=\sum_{i}^{3 N-6} M_{1 i}^{\alpha,|\omega|} \frac{\partial Q_{i}^{F}}{\partial F_{\alpha}^{|\omega|}}=\frac{\partial \phi_{1}^{F}}{\partial F_{\alpha}^{|\omega|}}=\frac{\partial \chi_{1}^{F}}{\partial F_{\alpha}^{|\omega|}},
$$

and, from the properties of an orthogonal matrix,

$$
\sum_{i}^{3 N-6} M_{j i}^{\alpha,|\omega|} M_{1 i}^{\alpha,|\omega|}=0 \quad \text { for } j>1
$$

Finally, using Eqs. (13)-(18) and the chain rule in the expression for $\alpha_{\alpha \beta}^{\mathrm{nr}}(\omega ; \omega)$ [i.e., Eq. (11)] we obtain

$$
\begin{aligned}
\alpha_{\alpha \beta}^{\mathrm{nr}}(\omega ; \omega) & =\frac{1}{2} \sum P_{\alpha \beta} \sum_{i}^{3 N-6} a_{11}^{i, \alpha} q_{1,|\omega|}^{i, \beta} \\
& =\frac{1}{2} \sum P_{\alpha \beta} \sum_{i}^{3 N-6} \frac{\partial \mu_{\alpha}}{\partial Q_{i}} \frac{\partial Q_{i}^{F}}{\partial F_{\beta}^{|\omega|}} \\
& =\frac{1}{2} \sum P_{\alpha \beta} \sum_{j}^{3 N-6} \frac{\partial \mu_{\alpha}}{\partial \phi_{j}^{\beta}} \sum_{i}^{3 N-6} M_{j i}^{\beta,|\omega|} M_{1 i}^{\beta,|\omega|} \\
& =\frac{1}{2} \sum P_{\alpha \beta} \frac{\partial \mu_{\alpha}}{\partial \chi_{1,|\omega|}^{\beta}} \frac{\partial \chi_{1}^{F}}{\partial F_{\beta}^{|\omega|}} .
\end{aligned}
$$

The above relation demonstrates that for $\alpha_{\alpha \beta}^{\mathrm{nr}}(\omega ; \omega)$ a sum over two appropriately chosen FD-FICs is completely equivalent to the usual sum over all $3 N-6$ normal coordinates. It should be clear that for the static case this is exactly the same result as found in Ref. 23.

Following a similar procedure it is easy to show that for $\beta_{\alpha \beta \gamma}^{\mathrm{nr}}\left(-\omega_{\sigma} ; \omega_{1}, \omega_{2}\right)$ three FICs are sufficient to reproduce the $3 N-6$ normal coordinate result. As in the previous demonstration we begin with the $\mathrm{BK} 3 N-6$ normal coordinate equation,

$$
\begin{aligned}
\beta_{\alpha \beta \gamma}^{\mathrm{nr}}\left(-\omega_{\sigma} ; \omega_{1}, \omega_{2}\right)= & \sum P_{\alpha \beta \gamma}\left[\sum_{i}^{3 N-6} a_{12}^{i, \alpha \beta} q_{1,\left|\omega_{2}\right|}^{i, \gamma}\right. \\
& -\sum_{i j}^{3 N-6} a_{21}^{i j, \alpha} q_{1,\left|\omega_{1}\right|}^{i, \beta} q_{1,\left|\omega_{2}\right|}^{j, \gamma} \\
& \left.+\sum_{i j k}^{3 N-6} a_{30}^{i j k} q_{1,\left|-\omega_{\sigma}\right|}^{i, \alpha} q_{1,\left|\omega_{1}\right|}^{j, \beta} q_{1,\left|\omega_{2}\right|}^{k, \gamma}\right],
\end{aligned}
$$

where $\sum P_{\alpha \beta \gamma}$ indicates a sum over all six permutations of the pairs of indices $(-\sigma / \alpha),(1 / \beta)$, and $(2 / \gamma)$. But, in this instance, introduce three sets of vibrational coordinates defined either by $\phi_{1}^{\alpha}=\chi_{1, \mid-\omega_{\sigma \mid}}^{\alpha}$ or $\phi_{1}^{\beta}=\chi_{1,\left|\omega_{1}\right|}^{\beta}$ or $\phi_{1}^{\gamma}=\chi_{1,\left|\omega_{2}\right|}^{\gamma}$ and their orthogonal complements. For instance, the second term on the rhs of Eq. (20) leads to

$$
\begin{aligned}
-\sum & P_{\alpha \beta \gamma} \sum_{i j}^{3 N-6} a_{21}^{i j, \alpha} q_{1,\left|\omega_{1}\right| q_{1,\left|\omega_{2}\right|}^{i, \beta}}^{j, \gamma} \\
= & \sum P_{\alpha \beta \gamma} \frac{1}{2} \sum_{i j}^{3 N-6} \frac{\partial^{2} \mu_{\alpha}}{\partial Q_{i} \partial Q_{j}} \frac{\partial Q_{i}^{F}}{\partial F_{\beta}^{\left|\omega_{1}\right|}} \frac{\partial Q_{j}^{F}}{\partial F_{\gamma}^{\left|\omega_{2}\right|}} \\
= & \sum P_{\alpha \beta \gamma} \frac{1}{2} \sum_{k l}^{3 N-6} \frac{\partial^{2} \mu_{\alpha}}{\partial \phi_{k}^{\beta} \partial \phi_{l}^{\gamma}} \sum_{i}^{3 N-6} M_{k i}^{\beta,\left|\omega_{1}\right|} M_{1 i}^{\beta,\left|\omega_{1}\right|} \\
& \times \sum_{j}^{3 N-6} M_{l j}^{\gamma,\left|\omega_{2}\right|} M_{1 j}^{\gamma,\left|\omega_{2}\right|} \\
= & \sum P_{\alpha \beta \gamma} \frac{1}{2} \frac{\partial^{2} \mu_{\alpha}}{\partial \chi_{1,\left|\omega_{1}\right|}^{\beta} \chi_{1,\left|\omega_{2}\right|}^{\gamma}} \frac{\partial \chi_{1}^{F}}{\partial F_{\beta}^{\left|\omega_{1}\right|}} \frac{\partial \chi_{1}^{F}}{\partial F_{\gamma}^{\left|\omega_{2}\right|}} .
\end{aligned}
$$

Applying the same procedure to the other two terms of $\beta_{\alpha \beta \gamma}^{\mathrm{nr}}\left(-\omega_{\sigma} ; \omega_{1}, \omega_{2}\right)$ one obtains ${ }^{24}$

$$
\begin{aligned}
& \beta_{\alpha \beta \gamma}^{\mathrm{nr}}\left(-\omega_{\sigma} ; \omega_{1}, \omega_{2}\right) \\
& =\sum P_{\alpha \beta \gamma} \\
& \times\left[\frac{1}{2} \frac{\partial \alpha_{\alpha \beta}}{\partial \chi_{1,\left|\omega_{2}\right|}^{\gamma}} \frac{\partial \chi_{1}^{F}}{\partial F_{\gamma}^{\left|\omega_{2}\right|}}+\frac{\partial^{2} \mu_{\alpha}}{\partial \chi_{1,\left|\omega_{1}\right|}^{\beta} \chi_{1,\left|\omega_{2}\right|}^{\gamma}} \frac{\partial \chi_{1}^{F}}{\partial F_{\beta}^{\left|\omega_{1}\right|}} \frac{\partial \chi_{1}^{F}}{\partial F_{\gamma}^{\left|\omega_{2}\right|}}\right. \\
& \left.-\frac{1}{6} \frac{\partial^{3} V}{\partial \chi_{1,\left|-\omega_{\sigma}\right|}^{\alpha} \chi_{1,\left|\omega_{1}\right|}^{\beta} \chi_{1,\left|\omega_{2}\right|}^{\gamma}} \frac{\partial \chi_{1}^{F}}{\partial F_{\alpha}^{\left|-\omega_{\sigma}\right|}} \frac{\partial \chi_{1}^{F}}{\partial F_{\beta}^{\mid \omega_{1}}} \frac{\partial \chi_{1}^{F}}{\partial F_{\gamma}^{\left|\omega_{2}\right|}}\right] .
\end{aligned}
$$

Here $\omega_{\sigma}=\sum_{i} \omega_{i}$. Since $\partial \chi_{1}^{F} / \partial F_{\alpha}^{|\omega|}$ vanishes in the limit $|\omega|$ $\rightarrow \infty$ it is easy to verify that Eq. (22) reduces to the expression given in an earlier work ${ }^{23}$ for $\beta_{z z z}^{\text {nr }}(-\omega ; \omega, 0)_{\omega \rightarrow \infty}$ and for $\beta_{z z z}^{\mathrm{nr}}(0 ; 0,0)$.

For $\gamma_{\alpha \beta \gamma \delta}^{\mathrm{nr}}\left(-\omega_{\sigma} ; \omega_{1}, \omega_{2}, \omega_{3}\right)$ we require second-order FD-FICs. These are obtained, as in the first-order case, by generalizing the static FIC expression. Thus, from Eq. (8),

$$
\chi_{2,\left|-\omega_{\sigma}+\omega_{1}\right|}^{\alpha \beta}=\frac{1}{2} \sum_{i=1}^{3 N-6} \frac{\partial^{2} Q_{i}^{F}}{\partial F_{\alpha}^{\left|-\omega_{\sigma}\right|} \partial F_{\beta}^{\left|\omega_{1}\right|}} Q_{i},
$$

where 


$$
\begin{aligned}
\frac{1}{2} \frac{\partial^{2} Q_{i}^{F}}{\partial F_{\alpha}^{\left|-\omega_{\sigma}\right|} \partial F_{\beta}^{\left|\omega_{1}\right|}}= & -q_{2,\left|-\omega_{\sigma}+\omega_{1}\right|}^{i, \alpha \beta} \\
& +\sum_{j=1}^{3 N-6} \frac{2 a_{2 l}^{i j, \alpha}}{2 a_{20}^{i i}-\left(-\omega_{\sigma}+\omega_{1}\right)^{2}} q_{1,\left|\omega_{1}\right|}^{j, \beta} \\
& -3 \sum_{j, k=1}^{3 N-6} \frac{a_{30}^{i j k}}{2 a_{20}^{i i}-\left(-\omega_{\sigma}+\omega_{1}\right)^{2}} \\
& \times q_{1,\left|-\omega_{\sigma}\right|}^{j, \alpha} q_{1,\left|\omega_{1}\right|}^{k, \beta}
\end{aligned}
$$

$$
q_{2,\left|-\omega_{\sigma}+\omega_{1}\right|}^{i, a \beta}=\frac{a_{12}^{i, a \beta}}{2 a_{20}^{i i}-\left(-\omega_{\sigma}+\omega_{1}\right)^{2}} .
$$

Proceeding as before we now need to introduce ten sets of FD-FICs (assuming all frequencies are different) based on $\chi_{1,\left|-\omega_{\sigma}\right|}^{\alpha}, \chi_{1,\left|\omega_{1}\right|}^{\beta}, \chi_{1,\left|\omega_{2}\right|}^{\gamma}, \chi_{1,\left|\omega_{3}\right|}^{\delta}, \chi_{2,\left|-\omega_{\sigma}+\omega_{1}\right|}^{\alpha \beta}, \chi_{2,\left|-\omega_{\sigma}+\omega_{2}\right|}^{\alpha \gamma}$, $\chi_{2,\left|-\omega_{\sigma}+\omega_{3}\right|}^{\alpha \delta}, \chi_{2,\left|\omega_{1}+\omega_{2}\right|}^{\beta \gamma}, \chi_{2,\left|\omega_{1}+\omega_{3}\right|}^{\beta \delta}$, and $\chi_{2,\left|\omega_{2}+\omega_{3}\right|}^{\gamma \delta}$. Substituting into the formula for $\gamma^{\mathrm{nr}}\left(-\omega_{\sigma} ; \omega_{1}, \omega_{2}, \omega_{3}\right)$ which can be written from the square bracket BK terms given in Ref. 20 yields $^{24}$

$$
\begin{aligned}
& \gamma_{\alpha \beta \gamma \delta}^{\mathrm{nr}}\left(-\omega_{\sigma} ; \omega_{1}, \omega_{2}, \omega_{3}\right)=\sum P_{\alpha \beta \gamma \delta}\left[\sum_{i}^{3 N-6}\left(a_{13}^{i, \alpha \beta \gamma} q_{1,\left|\omega_{3}\right|}^{i, \delta}+\frac{a_{12}^{i, \alpha \beta}}{2} a_{2,\left|\omega_{2}+\omega_{3}\right|}^{i, \gamma \delta}\right)-\sum_{i, j}^{3 N-6} a_{22}^{i j, \alpha \beta} q_{1,\left|\omega_{2}\right| q_{1,\left|\omega_{3}\right|}^{i, \gamma},{ }^{j, \delta}}^{3 N}\right. \\
& -2 \sum_{i, j}^{3 N-6} a_{21}^{i j, \alpha} q_{1,\left|\omega_{1}\right|}^{i, \beta} q_{2,\left|\omega_{2}+\omega_{3}\right|}^{j, \gamma \delta}+\sum_{i, j, k}^{3 N-6}\left(a_{31}^{i j k, \alpha} q_{1,\left|\omega_{1}\right|}^{i, \beta} q_{1,\left|\omega_{2}\right|}^{j, \gamma} q_{1,\left|\omega_{3}\right|}^{k, \delta}\right. \\
& +3 a_{30}^{i j k} q_{1, \mid-\omega_{\sigma}}^{i, \alpha} q_{1,\left|\omega_{1}\right|}^{j, \beta} q_{2,\left|\omega_{2}+\omega_{3}\right|}^{k, \gamma \delta}+\sum_{i, j, k}^{3 N-6} \frac{2 a_{21}^{i j, \alpha} a_{21}^{j k, \beta}}{2 a_{20}^{j j}-\left(-\omega_{\sigma}+\omega_{2}\right)^{2}} q_{1,\left|\omega_{2}\right|}^{i, \gamma} q_{1,\left|\omega_{3}\right|}^{k, \delta} \\
& -\sum_{i, j, k, l}^{3 N-6} a_{40}^{i j k l} q_{1,\left|-\omega_{\sigma}\right|}^{i, \alpha} q_{1,\left|\omega_{1}\right|}^{j, \beta} q_{1,\left|\omega_{2}\right|}^{k, \gamma} q_{1,\left|\omega_{3}\right|}^{l, \delta}-\sum_{i, j, k, l}^{3 N-6} \frac{6 a_{30}^{i j k} a_{21}^{k l, \alpha}}{2 a_{20}^{k k}-\left(-\omega_{\sigma}+\omega_{3}\right)^{2}} q_{1,\left|\omega_{1}\right|}^{i, \beta} q_{1,\left|\omega_{2}\right|}^{j, \gamma} q_{1,\left|\omega_{3}\right|}^{l, \delta} \\
& \left.+\frac{9}{2} \sum_{i, j, k, l, m}^{3 N-6} \frac{a_{30}^{i j k} a_{30}^{k l m}}{2 a_{20}^{k k}-\left(-\omega_{\sigma}+\omega_{1}\right)^{2}} q_{1, \mid-\omega_{\sigma}}^{i, \alpha} q_{1,\left|\omega_{1}\right|}^{j, \beta} q_{1,\left|\omega_{2}\right|}^{l, \gamma} q_{1,\left|\omega_{3}\right|}^{m, \delta}\right] \\
& =\sum P_{\alpha \beta \gamma \delta}\left[\frac{1}{6} \frac{\partial \beta_{\alpha \beta \gamma}}{\partial \chi_{1,\left|\omega_{3}\right|}^{\delta}} \frac{\partial \chi_{1}^{F}}{\partial F_{\delta}^{\left|\omega_{3}\right|}}+\frac{1}{8} \frac{\partial \alpha_{\alpha \beta}}{\partial \chi_{2,\left|\omega_{2}+\omega_{3}\right|}^{\gamma \delta}} \frac{\partial^{2} \chi_{2}^{F}}{\partial F_{\gamma}^{\left|\omega_{2}\right|} \partial F_{\delta}^{\left|\omega_{3}\right|}}+\frac{1}{4} \frac{\partial^{2} \alpha_{\alpha \beta}}{\partial \chi_{1,\left|\omega_{2}\right|}^{\gamma} \chi_{1,\left|\omega_{3}\right|}^{\delta}} \frac{\partial \chi_{1}^{F}}{\partial F_{\gamma}^{\left|\omega_{2}\right|}} \frac{\partial \chi_{1}^{F}}{\partial F_{\delta}^{\left|\omega_{3}\right|}}\right. \\
& +\frac{1}{4} \frac{\partial^{2} \mu_{\alpha}}{\partial \chi_{1,\left|\omega_{1}\right|}^{\beta} \partial \chi_{2,\left|\omega_{2}+\omega_{3}\right|}^{\gamma \delta}} \frac{\partial \chi_{1}^{F}}{\partial F_{\beta}^{\left|\omega_{1}\right|}} \frac{\partial^{2} \chi_{2}^{F}}{\partial F_{\gamma}^{\left|\omega_{2}\right|} \partial F_{\delta}^{\mid \omega_{3}}}+\frac{1}{6} \frac{\partial^{3} \mu_{\alpha}}{\partial \chi_{1,\left|\omega_{1}\right|}^{\beta} \partial \chi_{1,\left|\omega_{2}\right|}^{\gamma} \chi_{1,\left|\omega_{3}\right|}^{\delta}} \frac{\partial \chi_{1}^{F}}{\partial F_{\beta}^{\mid \omega_{1}}} \frac{\partial \chi_{1}^{F}}{\partial F_{\gamma}^{\mid \omega_{2}}} \frac{\partial \chi_{1}^{F}}{\partial F_{\delta}^{\left|\omega_{3}\right|}} \\
& -\frac{1}{8} \frac{\partial_{3} V}{\partial \chi_{1,\left|-\omega_{\sigma}\right|}^{\alpha} \chi_{1,\left|\omega_{1}\right|}^{\beta} \chi_{2,\left|\omega_{2}+\omega_{3}\right|}^{\gamma \delta}} \frac{\partial \chi_{1}^{F}}{\partial F_{\alpha}^{\left|-\omega_{\sigma}\right|}} \frac{\partial \chi_{1}^{F}}{\partial F_{\beta}^{\mid \omega_{1}}} \frac{\partial^{2} \chi_{2}^{F}}{\partial F_{\gamma}^{\left|\omega_{2}\right|} \partial F_{\delta}^{\left|\omega_{3}\right|}}
\end{aligned}
$$

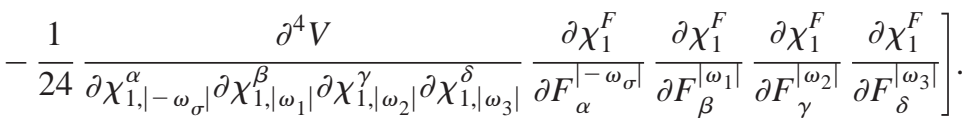

Since $\partial \chi_{1}^{F} / \partial F_{\alpha}^{|\omega|}$ vanishes in the limit $|\omega| \rightarrow \infty$; the harmonic and anharmonic parts of $\partial^{2} \chi_{2}^{F} / \partial F_{\gamma}^{\left|\omega_{i}\right|} \partial F_{\delta}^{\left|\omega_{j}\right|}$ vanish in the limit $\left|\omega_{i}+\omega_{j}\right| \rightarrow \infty$; and the anharmonic part of $\partial^{2} \chi_{2}^{F} / \partial F_{\gamma}^{\left|\omega_{i}\right|} \partial F_{\delta}^{\left|\omega_{j}\right|}$ vanishes if $\left|\omega_{i}\right| \rightarrow \infty$ and/or $\left|\omega_{j} \rightarrow \infty\right|$; it is easy to verify that this general expression reduces to what has been found for the special cases considered previously. ${ }^{23}$

Equations (19), (22), and (26) are the key results of our treatment. In the study of NLO materials one is often interested in only one or, perhaps, a few components of the (hyper)polarizability tensor. In that event the number of unique FD-FICs is reduced. For example, the longitudinal component of $\gamma_{\alpha \beta \gamma \delta}^{\mathrm{nr}}\left(-\omega_{\sigma} ; \omega_{1}, \omega_{2}, \omega_{3}\right)$, for which $\alpha=\beta=\gamma=\delta$ $=L$, is completely determined by seven FD-FICs: $\chi_{1,\left|-\omega_{\sigma}\right|}^{L}$,
$\chi_{1,\left|\omega_{1}\right|}^{L}, \chi_{1,\left|\omega_{2}\right|}^{L}, \chi_{1,\left|\omega_{3}\right|}^{L}, \chi_{2,\left|\omega_{1}+\omega_{2}\right|}^{L}, \chi_{2,\left|\omega_{1}+\omega_{3}\right|}^{L}$, and $\chi_{2,\left|\omega_{2}+\omega_{3}\right|}^{L}$ rather than ten. If, in addition, some of the optical frequencies are duplicated, then a further reduction occurs. In the case of the Kerr effect (i.e., $\omega_{\sigma}=\omega_{1}=\omega ; \omega_{2}=\omega_{3}=0$ ), for instance, there remain only two unique first-order FD-FICs $\left(\chi_{1,|0|}^{L}, \chi_{1,|\omega|}^{L}\right)$ and two unique second-order FICs $\left(\chi_{2,|0|}^{L}, \chi_{2,|\omega|}^{L}\right)$ for a total of four. In the static limit only one first-order and one second-order FIC $\left(\chi_{1,|0|}^{L}, \chi_{2,|0|}^{L}\right)$ endure and they are the same as those obtained in our earlier treatment. ${ }^{23}$ In fact, as we have already noted, it is straightforward to verify that, in the static limit, our key formulas for the (hyper)polarizabilities are identical to those found previously. 
TABLE I. Structural formula of molecules studied in this paper.

Number

The second-order FD-FIC defined by Eq. (24) contains two anharmonic terms which are tedious to calculate. In the static case these terms can be obtained by finite field geometry optimization but that is not possible when frequency dependence is taken into account. On the other hand, the harmonic approximation,

$$
\begin{aligned}
\chi_{2,\left|-\omega_{\sigma}+\omega_{1}\right|, \text { har }}^{\alpha \beta} & =\frac{1}{2} \sum_{i=1}^{3 N-6}\left(\frac{\partial^{2} Q_{i}^{\theta}}{\partial F_{\alpha}^{\left|-\omega_{\sigma}\right|} \partial F_{\beta}^{\left|\omega_{1}\right|}}\right)_{\text {har }} Q_{i} \\
& =-\sum_{i=1}^{3 N-6} q_{2, \mid-\omega_{\sigma}+\omega_{1}}^{i, \alpha \beta} Q_{i}
\end{aligned}
$$

is sufficient to obtain most properties either in the static or infinite optical frequency limit with the notable exception of the static $\gamma^{\mathrm{nr}}$. For the latter property using the harmonic approximation causes errors of second-order in anharmonicity. Since these second-order anharmonicity contributions are expected to fall off rapidly with increasing frequency the harmonic approximation is an attractive possibility for frequencies beyond the infrared region. In the next section we will explore that possibility along with other test calculations to establish the circumstances under which static FICs are insufficient and the frequency-dependent version is required.

\section{TEST CALCULATIONS}

This section has several goals with respect to the calculation of NR properties; (i) to examine the limits of the infinite frequency approximation; (ii) to determine when substitution of static FICs into the frequency-dependent BK perturbation formulas is adequate to describe frequency dispersion; (iii) in instances where (ii) is inadequate, to quantify the performance of various subsets of the complete set of FD-FICs; and, in conjunction with that study; (iv) to investigate the validity of the harmonic approximation for secondorder FICs. For initial testing purposes ab initio RHF/6-31G calculations were carried out on (see Table I) p-methylpyridone (I), 1-amino-4-nitrobuta-1,3-diene (II), and hexatriene (III). These three organic molecules are each representative of a different class of compound in terms of polarity and valence bond-charge transfer (VB-CT) characteristics; ${ }^{30} \mathrm{I}$ is polar with a dominant CT ground state; II is polar with a dominant VB ground state; and III is nonpolar.

The infinite optical frequency approximation has been found to perform satisfactorily for small molecules by Bishop and Dalskov ${ }^{20}$ as well as by Quinet and Champagne. ${ }^{21}$ However the behavior of this approximation for medium size organic molecules has not previously been tested. In Tables II-IV we present results for the frequencydependent NR contribution to $\alpha_{z z}(-\omega ; \omega), \beta_{z z z}(-\omega ; \omega, 0)$, $\beta_{z z z}(-2 \omega ; \omega, \omega), \quad \gamma_{z z z z}(-\omega ; \omega, 0,0), \quad \gamma_{z z z z}(-2 \omega ; \omega, \omega, 0)$, $\gamma_{z z z z}(-3 \omega ; \omega, \omega, \omega)$, and $\gamma_{z z z z}(-\omega ; \omega,-\omega, \omega)$ in molecules I-III, where $z$ is the (longitudinal) direction associated with the largest component of the (hyper)polarizability tensor. In our calculations we took $z$ to be along the principal axis associated with the largest rotational constant. For comparison purposes we also give the static electronic property value. The properties in Tables II-IV were evaluated at the Hartree-Fock 6-31G (HF/6-31G) level using the derivative expressions of Eqs. (19), (20), and (26). Although a higher level treatment is needed for accurate results, the calculations

TABLE II. Exact frequency-dependent NR (hyper)polarizabilities $\alpha_{z z}(-\omega ; \omega), \beta_{z z z}(-\omega ; \omega, 0)$, and $\beta_{z z z}(-2 \omega ; \omega, \omega)$ calculated at the HF/6-31G level using BK perturbation theory. Molecule I is p-methylpyridone; II is 1-amino-4-nitrobuta-1,3-diene; and III is hexatriene. The percentage error of the infinite optical frequency NR values with respect to the sum of the electronic and infinite optical frequency NR values are given in parentheses. Those cases where the error

\begin{tabular}{|c|c|c|c|c|c|c|c|}
\hline \multirow[b]{2}{*}{$\omega$} & \multicolumn{3}{|c|}{$\alpha_{z \bar{z}}(-\omega ; \omega)$} & \multicolumn{2}{|c|}{$\beta_{z z z}(-\omega ; \omega, 0)$} & \multicolumn{2}{|c|}{$\beta_{z z \bar{z}}(-2 \omega ; \omega, \omega)$} \\
\hline & I & II & III & I & II & I & II \\
\hline elec. & $1.03 \times 10^{2}$ & $1.39 \times 10^{2}$ & $1.42 \times 10^{2}$ & $-3.31 \times 10^{2}$ & $1.74 \times 10^{3}$ & $-3.31 \times 10^{2}$ & $1.74 \times 10^{3}$ \\
\hline 0.000 & $1.47 \times 10^{1}(14.3)$ & $4.03 \times 10^{1}(29.0)$ & $3.13 \times 10^{0}(2.2)$ & $2.44 \times 10^{2}(-66.7)$ & $4.88 \times 10^{3}(131.0)$ & $2.44 \times 10^{2}(-73.6)$ & $4.88 \times 10^{3}(280.0)$ \\
\hline 0.020 & $-2.22 \times 10^{0}(-2.2)$ & $-6.66 \times 10^{\circ}(-4.6)$ & $-2.45 \times 10^{-1}(-0.2)$ & $6.20 \times 10^{1}(2.6)$ & $5.83 \times 10^{2}(-18.9)$ & $-1.01 \times 10^{1}(3.0)$ & $-4.25 \times 10^{2}(-24.4)$ \\
\hline 0.040 & $-4.71 \times 10^{-1}(-0.5)$ & $-1.26 \times 10^{\circ}(-0.9)$ & $-3.76 \times 10^{-2}(0.0)$ & $6.58 \times 10^{1}(1.1)$ & $1.02 \times 10^{3}(-3.8)$ & $-3.31 \times 10^{0}(1.0)$ & $-8.56 \times 10^{1}(-4.9)$ \\
\hline 0.060 & $-2.04 \times 10^{-1}(-0.2)$ & $-5.48 \times 10^{-1}(-0.4)$ & $-1.58 \times 10^{-2}(0.0)$ & $6.74 \times 10^{1}(0.5)$ & $1.08 \times 10^{3}(-1.6)$ & $-1.48 \times 10^{\circ}(0.4)$ & $-3.73 \times 10^{1}(-2.1)$ \\
\hline 0.072 & $-1.41 \times 10^{-1}(-0.1)$ & $-3.78 \times 10^{-1}(-0.3)$ & $-1.08 \times 10^{-2}(0.0)$ & $6.78 \times 10^{1}(0.4)$ & $1.09 \times 10^{3}(-1.1)$ & $-1.03 \times 10^{0}(0.3)$ & $-2.58 \times 10^{1}(-1.5)$ \\
\hline 0.080 & $-1.14 \times 10^{-1}(-0.1)$ & $-3.06 \times 10^{-1}(-0.2)$ & $-8.71 \times 10^{-3}(0.0)$ & $6.80 \times 10^{1}(0.3)$ & $1.10 \times 10^{3}(-0.9)$ & $-8.35 \times 10^{-1}(0.3)$ & $-2.09 \times 10^{1}(-1.2)$ \\
\hline 0.100 & $-7.26 \times 10^{-2}(-0.1)$ & $-1.95 \times 10^{-1}(-0.1)$ & $-5.53 \times 10^{-3}(0.0)$ & $6.83 \times 10^{1}(0.2)$ & $1.11 \times 10^{3}(-0.6)$ & $-5.34 \times 10^{-1}(0.2)$ & $-1.33 \times 10^{1}(-0.8)$ \\
\hline 0.120 & $-5.03 \times 10^{-2}(-0.1)$ & $-1.35 \times 10^{-1}(-0.1)$ & $-3.82 \times 10^{-3}(0.0)$ & $6.84 \times 10^{1}(0.1)$ & $1.11 \times 10^{3}(-0.4)$ & $-3.71 \times 10^{-1}(0.1)$ & $-9.24 \times 10^{0}(-0.5)$ \\
\hline infinite & $0.00 \times 10^{0}$ & $0.00 \times 10^{\circ}$ & $0.00 \times 10^{0}$ & $6.88 \times 10^{1}$ & $1.12 \times 10^{3}$ & $0.00 \times 10^{0}$ & $0.00 \times 10^{0}$ \\
\hline
\end{tabular}
exceeds $5 \%$ are blocked off with dashed lines. All quantities are in a.u. 
TABLE III. Exact frequency-dependent NR hyperpolarizabilities $\gamma_{z z z z}(-\omega ; \omega, 0,0)$ and $\gamma_{z z z z}(-2 \omega ; \omega, \omega, 0)$ calculated at the HF/6-31G level using BK perturbation theory. Molecule I is p-methylpyridone; II is 1-amino-4-nitrobuta-1,3-diene; and III is hexatriene. The percentage error of the infinite optical frequency NR values with respect to the sum of the electronic and infinite optical frequency NR values are given in parentheses. Those cases where the error exceeds $5 \%$ are blocked off with dashed lines. All quantities are in a.u.

\begin{tabular}{|c|c|c|c|c|c|c|}
\hline \multirow[b]{2}{*}{$\omega$} & \multicolumn{3}{|c|}{$\gamma_{z z z z}(-\omega ; \omega, 0,0)$} & \multicolumn{3}{|c|}{$\gamma_{z z z z}(-2 \omega ; \omega, \omega, 0)$} \\
\hline & I & II & III & I & II & III \\
\hline elec. & $1.67 \times 10^{3}$ & $8.36 \times 10^{4}$ & $5.45 \times 10^{4}$ & $1.67 \times 10^{3}$ & $8.36 \times 10^{4}$ & $-5.45 \times 10^{4}$ \\
\hline 0.000 & $6.65 \times 10^{3}(-705,1)$ & $6.33 \times 10^{5}(233.7)$ & $9.14 \times 10^{4}(73.7)$ & $6.65 \times 10^{3}(-607.8)$ & $6.33 \times 10^{5}(604.5)$ & $9.14 \times 10^{4}(170.0)$ \\
\hline 0.020 & $-2.80 \times 10^{3}(-18,3)$ & $8.36 \times 10^{4}(-22.1)$ & $1.78 \times 10^{4}(-13.9)$ & $-3.20 \times 10^{3}(-7.1)$ & $-1.17 \times 10^{4}(-29.3)$ & $-1,32 \times 10^{4}(-23.5)$ \\
\hline 0.040 & $-2.95 \times 10^{3}(-7.5)$ & $1.21 \times 10^{5}(-4.8)$ & $2.71 \times 10^{4}(-2.9)$ & $-3.22 \times 10^{3}(-5.3)$ & $1.17 \times 10^{4}(-6.3)$ & $-3.10 \times 10^{3}(-4.9)$ \\
\hline 0.060 & $-3.00 \times 10^{3}(-3.3)$ & $1.27 \times 10^{5}(-2.1)$ & $2.85 \times 10^{4}(-1.2)$ & $-3.27 \times 10^{3}(-2.5)$ & $1.53 \times 10^{4}(-2.8)$ & $-1.59 \times 10^{3}(-2.1)$ \\
\hline 0.072 & $-3.02 \times 10^{3}(-2.3)$ & $1.28 \times 10^{5}(-1.4)$ & $2.88 \times 10^{4}(-0.9)$ & $-3.28 \times 10^{3}(-1.7)$ & $1.61 \times 10^{4}(-1.9)$ & $-1.24 \times 10^{3}(-1.5)$ \\
\hline 0.080 & $-3.02 \times 10^{3}(-1.9)$ & $1.29 \times 10^{5}(-1.2)$ & $2.90 \times 10^{4}(-0.7)$ & $-3.29 \times 10^{3}(-1.4)$ & $1.65 \times 10^{4}(-1.6)$ & $-1.08 \times 10^{3}(-1.2)$ \\
\hline 0.100 & $-3.03 \times 10^{3}(-1.2)$ & $1.29 \times 10^{5}(-0.7)$ & $2.92 \times 10^{4}(-0.4)$ & $-3.30 \times 10^{3}(-0.9)$ & $1.71 \times 10^{4}(-1.0)$ & $-8.51 \times 10^{2}(-0.8)$ \\
\hline 0.120 & $-3.04 \times 10^{3}(-0.8)$ & $1.30 \times 10^{5}(-0.5)$ & $2.93 \times 10^{4}(-0.3)$ & $-3.30 \times 10^{3}(-0.6)$ & $1.74 \times 10^{4}(-0.7)$ & $-7.25 \times 10^{2}(-0.5)$ \\
\hline infinite & $-3.05 \times 10^{3}$ & $1.31 \times 10^{5}$ & $2.95 \times 10^{4}$ & $-3.31 \times 10^{3}$ & $1.81 \times 10^{4}$ & $-4.42 \times 10^{2}$ \\
\hline
\end{tabular}

reported are sufficient for the exploratory purposes to which they have been put here.

For our three molecules it is feasible to use the GAUSSIAN 98 suite of programs ${ }^{31}$ to obtain analytical HF/6$31 \mathrm{G}$ results for $a_{20}, a_{01}, a_{11}, a_{02}, a_{12}$, and $a_{03}$ where the vibrational derivatives are with respect to atomic Cartesian coordinates. These derivatives may be combined to obtain derivatives with respect to normal coordinates or FD-FICs. Then, numerical differentiation of $a_{20}, a_{11}, a_{12}$, and $a_{03}$ with respect to the normal coordinates or FD-FICs yields $a_{30}, a_{21}, a_{22}$, and $a_{13}$, respectively. $a_{40}$ and $a_{31}$ were computed by double numerical differentiation of $a_{20}$ and $a_{11}$. The NR contributions that lie inside the dashed line area of the tables represent those cases where there is a significant difference between the frequency-dependent NR contribution and the infinite frequency approximation. As a measure of significance we have chosen to compare this difference to the sum of the static electronic property value and the infinite frequency NR contribution. The criterion for significance was arbitrarily set at $5 \%$. We note that the peculiar behavior in the tables between 0.00 and 0.02 a.u. arises because these frequencies lie in the vibrational absorption region.

For those properties that have a vanishing NR contribution under the infinite frequency approximation, i.e., $\alpha_{z z}(-\omega ; \omega), \quad \beta_{z z z}(-2 \omega ; \omega, \omega), \quad$ and $\quad \gamma_{z z z z}(-3 \omega ; \omega, \omega, \omega)$, the frequency-dependent NR term always tends rapidly to zero as $\omega$ increases. Tables II-IV show that for these properties the error is insignificant when $\omega \geqslant 0.04 \mathrm{a}$.u. The only exception is $\gamma_{z z z}^{\mathrm{nr}}(-3 \omega ; \omega, \omega, \omega)$ of molecule I, which has a small static electronic value and, in that case, the error becomes insignificant when $\omega \geqslant 0.08$ a.u. On the other hand, our results for $\beta_{z z z}^{\mathrm{nr}}(-\omega ; \omega, 0), \quad \gamma_{z z z z}^{\mathrm{nr}}(-\omega ; \omega, 0,0)$, $\gamma_{z z z z}^{\mathrm{nr}}(-2 \omega ; \omega ; \omega ; 0)$, and $\gamma_{z z z z}^{\mathrm{nr}}(-\omega ; \omega,-\omega, \omega)$ indicate, that for these properties, the infinite frequency approximation can not be systematically applied without significant error when the optical frequency is lower than $\omega=0.06$ a.u. $\left(\sim 13000 \mathrm{~cm}^{-1}\right)$. This is consistent with the theoretical assumption underpinning the infinite frequency approximation that $\left(\omega_{v} / \omega\right)^{2}$, with $\omega_{v}$ a fundamental vibrational frequency, is negligible compared to unity.

There is a way to introduce frequency-dependence using only the static FICs and that is to substitute these FICs for the normal coordinates directly into the usual frequencydependent perturbation formulas. For the polarizability and first hyperpolarizability only the first-order static FIC is utilized. For the second hyperpolarizability the second-order FIC is required as well. In that case there are two possibili-

TABLE IV. Exact frequency-dependent NR hyperpolarizabilities $\gamma_{z z z z}(-3 \omega ; \omega, \omega, \omega)$ and $\gamma_{z z z z}(-\omega ; \omega,-\omega, \omega)$ calculated at the HF/6-31G level using BK perturbation theory. Molecule I is p-methylpyridone; II is 1-amino-4-nitrobuta-1,3-diene; and III is hexatriene. The percentage error of the infinite optical frequency NR values with respect to the sum of the electronic and infinite optical frequency NR values are given in parentheses. Those cases where the error exceeds $5 \%$ are blocked off with dashed lines. All quantities are in a.u.

\begin{tabular}{|c|c|c|c|c|c|c|}
\hline \multirow[b]{2}{*}{$\omega$} & \multicolumn{3}{|c|}{$\gamma_{z z z z}(-3 \omega ; \omega, \omega, \omega)$} & \multicolumn{3}{|c|}{$\gamma_{z z z z}(-\omega ; \omega,-\omega, \omega)$} \\
\hline & I & II & III & I & II & III \\
\hline elec. & $1.67 \times 10^{3}$ & $8.36 \times 10^{4}$ & $5.45 \times 10^{4}$ & $1.67 \times 10^{3}$ & $8.36 \times 10^{4}$ & $5.45 \times 10^{4}$ \\
\hline 0.000 & $6.65 \times 10^{3}(397.3)$ & $6.33 \times 10^{5}(757,0)$ & $9.14 \times 10^{4}(167.8)$ & $6.65 \times 10^{3}(11.6)$ & $6.33 \times 10^{5}(316.3)$ & $9.14 \times 10^{4}(27.9)$ \\
\hline 0.020 & $1.38 \times 10^{3}(82.2)$ & $-1.21 \times 10^{4}(-14.4)$ & $-3.41 \times 10^{3}(-6.2)$ & $7.84 \times 10^{3}(27.7)$ & $5.92 \times 10^{4}(-17.0)$ & $5.85 \times 10^{4}(-1.0)$ \\
\hline 0.040 & $3.09 \times 10^{2}(18.5)$ & $-2.75 \times 10^{3}(-3.3)$ & $-8.25 \times 10^{2}(-1.5)$ & $6.22 \times 10^{3}(5.9)$ & $8.16 \times 10^{4}(-4.0)$ & $5.93 \times 10^{4}(-0.2)$ \\
\hline 0.060 & $1.35 \times 10^{2}(8.1)$ & $-1.22 \times 10^{3}(-1.5)$ & $-3.65 \times 10^{2}(-0.7)$ & $5.97 \times 10^{3}(2.5)$ & $8.55 \times 10^{4}(-1.8)$ & $5.95 \times 10^{4}(-0.1)$ \\
\hline 0.072 & $9.31 \times 10^{1}(5.6)$ & $-8.46 \times 10^{2}(-1.0)$ & $-2.53 \times 10^{2}(-0.5)$ & $5.91 \times 10^{3}(1.8)$ & $8.64 \times 10^{4}(-1.2)$ & $5.95 \times 10^{4}(-0.1)$ \\
\hline 0.080 & $7.53 \times 10^{1}(4.5)$ & $-6.85 \times 10^{2}(-0.8)$ & $-2.05 \times 10^{2}(-0.4)$ & $5.89 \times 10^{3}(1.4)$ & $8.68 \times 10^{4}(-1.0)$ & $5.96 \times 10^{4}(-0.1)$ \\
\hline 0.100 & $4.80 \times 10^{1}(2.9)$ & $-4.38 \times 10^{2}(-0.5)$ & $-1.31 \times 10^{2}(-0.2)$ & $5.85 \times 10^{3}(0.9)$ & $8.74 \times 10^{4}(-0.6)$ & $5.96 \times 10^{4}(0.0)$ \\
\hline 0.120 & $3.33 \times 10^{1}(2.0)$ & $-3.04 \times 10^{2}(-0.4)$ & $-9.08 \times 10^{1}(-0.2)$ & $5.83 \times 10^{3}(0.6)$ & $8.77 \times 10^{4}(-0.4)$ & $5.96 \times 10^{4}(0.0)$ \\
\hline infinite & $0.000 \times 10^{0}$ & $0.00 \times 10^{0}$ & $0.00 \times 10^{0}$ & $5.78 \times 10^{3}$ & $8.85 \times 10^{4}$ & $5.96 \times 10^{4}$ \\
\hline
\end{tabular}


TABLE V. Frequency-dependent NR (hyper)polarizabilities $\alpha_{z z}(-\omega ; \omega), \beta_{z z z}(-\omega, \omega, 0)$, and $\beta_{z z z}(-2 \omega ; \omega, \omega)$ calculated at the HF/6-31G level using theory and static FICs. Molecule I is $p$-methylpyridone; II is 1-amino-4-nitrobuta-1,3-diene; and III is hexatriene. The percentage error with respect to the sum of the electronic and exact dynamic NR values are given in parentheses. Those cases where the error exceeds $5 \%$ are blocked off with dashed lines. All quantities are in a.u.

\begin{tabular}{|c|c|c|c|c|c|c|c|}
\hline \multirow[b]{2}{*}{$\omega$} & \multicolumn{3}{|c|}{$\alpha_{z z}^{\mathrm{nr}}(-\omega ; \omega)$} & \multicolumn{2}{|c|}{$\beta_{z z z}^{\mathrm{nr}}(-\omega ; \omega, 0)$} & \multicolumn{2}{|c|}{$\beta_{z z z}^{\mathrm{nr}}(-2 \omega ; \omega, \omega)$} \\
\hline & I & $\Pi$ & III & I & $\Pi$ & I & II \\
\hline 0.000 & $1.47 \times 10^{1}(0.0)$ & $4.03 \times 10^{1}(0.0)$ & $3.13 \times 10^{0}(0.0)$ & $2.44 \times 10^{2}(0.0)$ & $4.88 \times 10^{3}(0.0)$ & $2.44 \times 10^{2}(0.0)$ & $4.88 \times 10^{3}(0.0)$ \\
\hline 0.020 & $-4.68 \times 10^{-1}(1.7)$ & $-3.60 \times 10^{0}(2.1)$ & $-6.03 \times 10^{-3}(0.2)$ & $6.79 \times 10^{1}(-2,2)$ & $8.38 \times 10^{2}(11.0)$ & $-8.24 \times 10^{-1}(-2.7)$ & $-2.19 \times 10^{2}(15.6)$ \\
\hline 0.040 & $-1.14 \times 10^{-1}(0.3)$ & $-8.43 \times 10^{-1}(0.3)$ & $-1.51 \times 10^{-3}(0.0)$ & $6.86 \times 10^{1}(-1.0)$ & $1.06 \times 10^{3}(1.5)$ & $-2.28 \times 10^{-1}(-0.9)$ & $-5.27 \times 10^{1}(2.0)$ \\
\hline 0.060 & $-5.05 \times 10^{-2}(0.1)$ & $-3.70 \times 10^{-1}(0.1)$ & $-6.63 \times 10^{-4}(0.0)$ & $6.87 \times 10^{1}(-0.5)$ & $1.09 \times 10^{3}(0.6)$ & $-1.03 \times 10^{-1}(-0.4)$ & $-2.33 \times 10^{1}(0.8)$ \\
\hline 0.072 & $-3.50 \times 10^{-2}(0.1)$ & $-2.56 \times 10^{-1}(0.1)$ & $-3.77 \times 10^{-4}(0.0)$ & $6.87 \times 10^{1}(-0.3)$ & $1.10 \times 10^{3}(0.4)$ & $-7.19 \times 10^{-2}(-0.3)$ & $-1.61 \times 10^{1}(0.6)$ \\
\hline 0.080 & $-2.84 \times 10^{-2}(0.1)$ & $-2.07 \times 10^{-1}(0.1)$ & $-4.65 \times 10^{-4}(0.0)$ & $6.87 \times 10^{1}(-0.3)$ & $1.11 \times 10^{3}(0.3)$ & $-5.83 \times 10^{-2}(-0.2)$ & $-1.31 \times 10^{1}(0.5)$ \\
\hline 0.100 & $-1.81 \times 10^{-2}(0.1)$ & $-1.33 \times 10^{-1}(0.0)$ & $-2.41 \times 10^{-4}(0.0)$ & $6.87 \times 10^{1}(-0.2)$ & $1.11 \times 10^{3}(0.2)$ & $-3.74 \times 10^{-2}(-0.1)$ & $-8.35 \times 10^{0}(0.3)$ \\
\hline 0.120 & $-1.26 \times 10^{-2}(0.0)$ & $-9.19 \times 10^{-2}(0.0)$ & $-1.67 \times 10^{-4}(0.0)$ & $6.88 \times 10^{1}(-0.1)$ & $1.12 \times 10^{3}(0.1)$ & $-2.60 \times 10^{-2}(-0.1)$ & $-5.79 \times 10^{0}(0.2)$ \\
\hline infinite & $0.00 \times 10^{0}(0.0)$ & $0.00 \times 10^{0}(0.0)$ & $0.00 \times 10^{0}(0.0)$ & $6.88 \times 10^{1}(0.0)$ & $1.12 \times 10^{3}(0.0)$ & $0.00 \times 10^{0}(0.0)$ & $0.00 \times 10^{0}(0.0)$ \\
\hline
\end{tabular}

ties. The complete second-order static FIC yields the exact static NR second hyperpolarizability but, for optical processes, the harmonic FIC is necessary to obtain the exact solution in the infinite frequency limit. Thus, the frequencydependent NR properties presented in Tables V-VII were calculated utilizing harmonic static FICs. Of course, these results are approximate for all optical processes at finite, nonzero frequencies. As in Tables II-IV, those results containing a significant $(>5 \%)$ error are marked off by dashed lines. In this case the percent error was calculated by comparing to the sum of the static electronic property and the exact NR contribution. Although substitution of static FICs into the perturbation expressions often improves upon the infinite frequency approximation, the extent of that improvement depends considerably on the property and the molecule. Errors are reduced between $28 \%$ for $\gamma_{z z z z}^{\mathrm{nr}}(-2 \omega ; \omega, \omega, 0)$ at 0.02 a.u. (i.e., from $29.3 \%$ to $21.2 \%$ ) and $68 \%$ for $\alpha_{z z}^{\mathrm{nr}}(-\omega ; \omega)$ at 0.12 a.u. $(-0.10 \%$ to $0.03 \%)$ in the case of molecule II, whereas the reduction is between $2.3 \%$ for $\alpha_{z z}^{\mathrm{nr}}(-\omega ; \omega)$ at 0.02 a.u. $(-0.172 \%$ to $0.168 \%)$ and $35.2 \%$ for $\gamma_{z z z z}^{\mathrm{nr}}(-3 \omega ; \omega, \omega, \omega)$ at 0.12 a.u. $(-0.17 \%$ to $0.11 \%)$ in the case of molecule III. For molecule I the error in $\gamma_{z z z z}^{\mathrm{nr}}(-\omega ; \omega, 0,0)$ and $\gamma_{z z z z}^{\mathrm{nr}}(-2 \omega ; \omega, \omega, 0)$ actually increases, although reductions up to $54 \%$ are achieved for the remaining properties. Since the improvement is limited, the above method is not that attractive over the infinite frequency ap- proximation, especially when one considers the necessity of determining higher-order derivatives. In order to calculate the dynamic NR contribution to the second hyperpolarizability it is necessary to determine fourth derivatives of the energy with respect to vibrational coordinates $\left(a_{40}\right)$ and third derivatives of the dipole moment $\left(a_{31}\right)$, neither of which occur in the infinite optical frequency approximation for the same quantities. If we use FD-FICs one still has to determine the higher-order derivatives but, then, the calculated results are exact as long as a complete set is employed (cf. Sec. II).

In connection with FD-FICs an interesting question is whether sufficient accuracy can be achieved with less than a complete set. In order to address that point we focus on the most problematic case, namely on the hyperpolarizabilities of molecule I at $\omega=0.02$ and 0.04 . In addition, we also carried out calculations at $\omega=0.005$ a.u. $\left(1097 \mathrm{~cm}^{-1}\right)$ in order to analyze the performance of the FD-FICs within the IR region. Apart from using just a subset of the second-order FD-FICs we also considered only the harmonic approximation to the second-order FD-FICs for the second hyperpolarizabilities. This considerably simplifies the calculations because, then, all numerical differentiations are done with respect to the FD-FICs. Otherwise, $a_{20}, a_{11}$, and $a_{12}$ must be differentiated with respect to the $3 N-6$ normal coordinates.

Our results for several subsets are given in the Tables

TABLE VI. Frequency-dependent NR hyperpolarizabilities, $\gamma_{z z z z}^{\mathrm{nr}}(-\omega ; \omega, 0,0)$ and $\gamma_{z z z z}^{\mathrm{nr}}(-2 \omega ; \omega, \omega, 0)$ calculated at the HF/6-31G level using BK perturbation theory and static FICs. Molecule I is p-methylpyridone; II is 1-amino-4-nitrobuta-1,3-diene; and III is hexatriene. The percentage error with respect to the sum of the electronic and exact dynamic NR values are given in parentheses. Those cases where the error exceeds $5 \%$ are blocked off with dashed lines. All quantities are in a.u.

\begin{tabular}{|c|c|c|c|c|c|c|}
\hline \multirow[b]{2}{*}{$\omega$} & \multicolumn{3}{|c|}{$\gamma_{z z Z z}^{\mathrm{nr}}(-\omega ; \omega, 0,0)$} & \multicolumn{3}{|c|}{$\gamma_{z z z z}^{\mathrm{nr}}(-2 \omega ; \omega, \omega, 0)$} \\
\hline & I & II & III & I & II & III \\
\hline 0.000 & $-3.24 \times 10^{2}(-83.8)$ & $6.32 \times 10^{5}(-0.1)$ & $8.64 \times 10^{4}(-3.4)$ & $-3.24 \times 10^{2}(-83.8)$ & $6.32 \times 10^{5}(-0.1)$ & $8.64 \times 10^{4}(-3.4)$ \\
\hline 0.020 & $-3.02 \times 10^{3}(19.9)$ & $1.06 \times 10^{5}(13.3)$ & $2.61 \times 10^{4}(11.4)$ & $-3.31 \times 10^{3}(7.6)$ & $3.55 \times 10^{3}(21.2)$ & $-4.20 \times 10^{3}(21.7)$ \\
\hline 0.040 & $-3.04 \times 10^{3}(7.6)$ & $1.25 \times 10^{5}(2.0)$ & $2.87 \times 10^{4}(2.0)$ & $-3,31 \times 10^{3}(5,6)$ & $1.45 \times 10^{4}(2.9)$ & $-1.35 \times 10^{3}(3.4)$ \\
\hline 0.060 & $-3.05 \times 10^{3}(3.3)$ & $1.28 \times 10^{5}(0.8)$ & $2.92 \times 10^{4}(0.8)$ & $-3.31 \times 10^{3}(2.5)$ & $1.65 \times 10^{4}(1.2)$ & $-8.42 \times 10^{2}(1.4)$ \\
\hline 0.072 & $-3.05 \times 10^{3}(2.3)$ & $1.29 \times 10^{5}(0.6)$ & $2.93 \times 10^{4}(0.6)$ & $-3.31 \times 10^{3}(1.8)$ & $1.70 \times 10^{4}(0.8)$ & $-7.19 \times 10^{2}(1.0)$ \\
\hline 0.080 & $-3.05 \times 10^{3}(1.8)$ & $1.30 \times 10^{5}(0.5)$ & $2.93 \times 10^{4}(0.5)$ & $-3.31 \times 10^{3}(1.4)$ & $1.72 \times 10^{4}(0.7)$ & $-6.66 \times 10^{2}(0.8)$ \\
\hline 0.100 & $-3.05 \times 10^{3}(1.2)$ & $1.30 \times 10^{5}(0.3)$ & $2.94 \times 10^{4}(0.3)$ & $-3.31 \times 10^{3}(0.9)$ & $1.75 \times 10^{4}(0.4)$ & $-5.86 \times 10^{2}(0.5)$ \\
\hline 0.120 & $-3.05 \times 10^{3}(0.8)$ & $1.30 \times 10^{5}(0.2)$ & $2.94 \times 10^{4}(0.2)$ & $-3.31 \times 10^{3}(0.6)$ & $1.77 \times 10^{4}(0.3)$ & $-5.42 \times 10^{2}(0.3)$ \\
\hline infinite & $-3.05 \times 10^{3}(0.0)$ & $1.31 \times 10^{5}(0.0)$ & $2.95 \times 10^{4}(0.0)$ & $-3.31 \times 10^{3}(0.0)$ & $1.81 \times 10^{4}(0.0)$ & $-4.42 \times 10^{2}(0.0)$ \\
\hline
\end{tabular}


TABLE VII. Frequency-dependent NR hyperpolarizabilities, $\gamma_{z z z z}^{\mathrm{nr}}(-3 \omega ; \omega, \omega, \omega)$ and $\gamma_{z z z z}^{\mathrm{nr}}(-\omega ; \omega,-\omega, \omega)$ calculated at the HF/6-31G level using BK perturbation theory and static FICs. Molecule I is p-methylpyridone; II is 1-amino-4-nitrobuta-1,3-diene; and III is hexatriene. The percentage error with respect to the sum of the electronic and exact dynamic NR values are given in parentheses. Those cases where the error exceeds 5\% are blocked off with dashed lines. All quantities are in a.u.

\begin{tabular}{|c|c|c|c|c|c|c|}
\hline \multirow[b]{2}{*}{$\omega$} & \multicolumn{3}{|c|}{$\gamma_{z z z z}^{\mathrm{nr}}(-3 \omega ; \omega, \omega, \omega)$} & \multicolumn{3}{|c|}{$\gamma_{z z z z}^{\mathrm{nr}}(-\omega ; \omega,-\omega, \omega)$} \\
\hline & I & II & III & I & II & III \\
\hline 0.000 & $-3.24 \times 10^{2}(-83.8)$ & $6.32 \times 10^{5}(-0.1)$ & $8.64 \times 10^{4}(-3.4)$ & $-3.24 \times 10^{2}(-83.8)$ & $6.32 \times 10^{5}(-0.1)$ & $8.64 \times 10^{4}(-3.4)$ \\
\hline 0.020 & $2.17 \times 10^{2}(-38.0)$ & $-6.62 \times 10^{3}(7.6)$ & $-1.17 \times 10^{3}(4.4)$ & $6.09 \times 10^{3}(-18.4)$ & $7.03 \times 10^{4}(7.8)$ & $5.92 \times 10^{4}(0.6)$ \\
\hline 0.040 & $5.29 \times 10^{1}(-12.9)$ & $-1.67 \times 10^{3}(1.3)$ & $-2.90 \times 10^{2}(1.0)$ & $5.86 \times 10^{3}(-4.6)$ & $8.40 \times 10^{4}(1.5)$ & $5.95 \times 10^{4}(0.2)$ \\
\hline 0.060 & $2.34 \times 10^{1}(-6.2)$ & $-7.45 \times 10^{2}(0.6)$ & $-1.28 \times 10^{2}(0.4)$ & $5.81 \times 10^{3}(-2.0)$ & $8.65 \times 10^{4}(0.6)$ & $5.96 \times 10^{4}(0.1)$ \\
\hline 0.072 & $1.62 \times 10^{1}(-4.4)$ & $-5.18 \times 10^{2}(0.4)$ & $-8.92 \times 10^{1}(0.3)$ & $5.80 \times 10^{3}(-1.4)$ & $8.71 \times 10^{4}(0.4)$ & $5.96 \times 10^{4}(0.0)$ \\
\hline 0.080 & $1.32 \times 10^{1}(-3.6)$ & $-4.19 \times 10^{2}(0.3)$ & $-7.22 \times 10^{1}(0.2)$ & $5.80 \times 10^{3}(-1.1)$ & $8.74 \times 10^{4}(0.3)$ & $5.96 \times 10^{4}(0.0)$ \\
\hline 0.100 & $8.41 \times 10^{0}(-2.3)$ & $-2.68 \times 10^{2}(0.2)$ & $-4.62 \times 10^{1}(0.2)$ & $5.79 \times 10^{3}(-0.7)$ & $8.78 \times 10^{4}(0.2)$ & $5.96 \times 10^{4}(0.0)$ \\
\hline 0.120 & $5.84 \times 10^{0}(-1.6)$ & $-1.86 \times 10^{2}(0.1)$ & $-3.21 \times 10^{1}(0.1)$ & $5.79 \times 10^{3}(-0.5)$ & $8.80 \times 10^{4}(0.2)$ & $5.96 \times 10^{4}(0.0)$ \\
\hline infinite & $0.00 \times 10^{0}(0.0)$ & $0.00 \times 10^{0}(0.0)$ & $0.00 \times 10^{0}(0.0)$ & $5.78 \times 10^{3}(0.0)$ & $8.85 \times 10^{4}(0.0)$ & $5.96 \times 10^{4}(0.0)$ \\
\hline
\end{tabular}

VIII and IX. We list the FD-FICs employed in the form $\left(\omega_{1}, \omega_{1}^{\prime}, \ldots / \omega_{2}, \omega_{2}^{\prime}, \ldots\right)$ where the first-order FICs are on the left of the diagonal line while the second-order FICs are on the right. For $\beta_{z z z}^{\mathrm{nr}}(-\omega ; \omega, 0)$ and $\gamma_{z z z z}^{\mathrm{nr}}(-\omega ; \omega, 0,0)$ the sets $(\omega, 0 / \omega, 0)$ and $(2 \omega, \omega, 0 / 2 \omega, \omega, 0)$ are both complete. Indeed, for either set our value of the first hyperpolarizability agrees with the exact result at all three frequencies as it should. The second hyperpolarizability, however, does not agree; the difference is due to the harmonic approximation for the second-order FICs. A similar analysis pertains to $\beta_{z z z}^{\mathrm{nr}}(-2 \omega ; \omega, \omega), \quad \gamma_{z z z z}^{\mathrm{nr}}(-2 \omega ; \omega, \omega, 0), \quad$ and $\quad \gamma_{z z z z}^{\mathrm{nr}}(-\omega ; \omega$, $-\omega, \omega)$. In this instance, although the set $(2 \omega, \omega, 0 / 2 \omega, \omega, 0)$ is complete $(\omega, 0 / \omega, 0)$ is not. (However, for all three properties there are smaller subsets not listed here that are complete.) The errors in the second hyperpolarizabilites that occur for the complete set again arise from the harmonic approximation for the second-order FICs. For $\alpha_{z z}^{\mathrm{nr}}(-\omega ; \omega)$ all of the FD-FIC sets chosen produce the exact value because they are all complete and we have, therefore, refrained from including this property in the table.
The most important result that emerges from Tables VIII and IX is that outside the IR region the set $(\omega, 0 / \omega, 0)$ is sufficient for all processes. This is true even in cases where this set is not complete and regardless of the fact that the harmonic approximation is applied for the second-order FICs. The first hyperpolarizabilities cannot be affected by the harmonic approximation. That is not true of the second hyperpolarizabilities but, in practice, $\gamma_{z z z z}^{\mathrm{nr}}(-\omega ; \omega, 0,0)$ is not significantly altered even within the IR region.

\section{CONCLUSIONS AND FUTURE WORK}

We have demonstrated that $\alpha_{\alpha \beta}^{\mathrm{nr}}\left(-\omega_{\sigma} ; \omega_{1}\right)$, $\beta_{\alpha \beta \gamma}^{\mathrm{nr}}\left(-\omega_{\sigma} ; \omega_{1}, \omega_{2}\right)$, and $\gamma_{\alpha \beta \gamma \delta}^{\mathrm{nr}}\left(-\omega_{\sigma} ; \omega_{1}, \omega_{2}, \omega_{3}\right)$ can be expressed exactly in terms of frequency-dependent fieldinduced vibrational coordinates called FD-FICs. The main advantage of the FD-FICs, as opposed to normal coordinates, is that they are few in number and the size of the set remains the same regardless of the number of atoms in the molecule. In previous work the (hyper)polarizabilities listed above

TABLE VIII. Frequency-dependent NR hyperpolarizabilities of $\beta_{z z z}(-\omega ; \omega, 0), \beta_{z z z}(-2 \omega ; \omega ; \omega)$ and $\gamma_{z z z z}^{\text {nr }}(-\omega ; \omega, 0,0)$ of $p$-methylpyridone calculated at the HF/6-31G level using BK perturbation theory and various sets of harmonic TD-FICs. The exact result is included for sake of comparison. The percentage error with respect to the sum of the electronic and exact dynamic NR values are given in parentheses. Those cases where the error exceeds 5\% are blocked off with dashed lines. All quantities are in a.u.

\begin{tabular}{|c|c|c|c|c|c|c|}
\hline$\omega$ & $(0 / 0)$ & $(\omega / \varnothing)$ & $(\omega / \omega)$ & $(\omega, 0 / \omega, 0)$ & $(2 \omega, \omega, 0 / 2 \omega, \omega, 0)$ & Exact \\
\hline \multicolumn{7}{|c|}{$\beta_{z z z}^{\mathrm{nr}}(-\omega ; \omega, 0)$} \\
\hline 0.005 & $1.42 \times 10^{2}(-320.4)$ & $3.15 \times 10^{2}(-118.1)$ & $3.56 \times 10^{2}(-71.3)$ & $4.17 \times 10^{2}(-0.1)$ & $4.17 \times 10^{2}(-0.1)$ & $4.17 \times 10^{2}$ \\
\hline 0,020 & $6.79 \times 10^{1}(-2.2)$ & $8.99 \times 10^{0}(19.7)$ & $4.80 \times 10^{1}(5.2)$ & $6.20 \times 10^{1}(0.0)$ & $6.20 \times 10^{1}(0,0)$ & $6.20 \times 10^{1}$ \\
\hline 0.040 & $6.86 \times 10^{1}(-1.0)$ & $2.91 \times 10^{1}(13.8)$ & $5.38 \times 10^{1}(4.5)$ & $6.58 \times 10^{1}(0.0)$ & $6.58 \times 10^{1}(0.0)$ & $6.58 \times 10^{1}$ \\
\hline
\end{tabular}

$\beta_{z z z}^{\mathrm{nt}}(-2 \omega ; \omega, \omega)$

0.005

0.020

0.040

$\begin{array}{ll}1.93 \times 10^{2}(-81.4) & -7.57 \times 10^{1}(-45.1) \\ -8.24 \times 10^{-1}(-2.7) & -9.45 \times 10^{0}(-0.2) \\ -2.28 \times 10^{-1}(-0.9) & -3.29 \times 10^{0}(0.0)\end{array}$

$-3.29 \times 10^{0}(0.0)$
$-1.11 \times 10^{2}(-40.3)$

$-1.01 \times 10^{1}(0.0)$

$-3.32 \times 10^{0}(0.0)$
$6.58 \times 10^{1}(0.0)$
$4.17 \times 10^{2}(-0.1)$
$6.20 \times 10^{1}(0.0)$

$6.58 \times 10^{1}(0.0)$

$\gamma_{z z z z}^{\mathrm{tII}}(-\omega ; \omega, 0,0)$

\begin{tabular}{|c|c|c|c|c|c|c|}
\hline 0.005 & $3.09 \times 10^{3}(-93.3)$ & $-4.51 \times 10^{3}(-104.0)$ & $3.60 \times 10^{4}(-46.8)$ & $6.54 \times 10^{4}(-5.4)$ & $6.58 \times 10^{4}(-4.8)$ & $6.92 \times 10^{4}$ \\
\hline 0.020 & $-3.02 \times 10^{3}(19.9)$ & $-2.92 \times 10^{3}(11.5)$ & $-4.24 \times 10^{3}(128.1)$ & $-2.79 \times 10^{3}(-0.1)$ & $-2.78 \times 10^{3}(-1.3)$ & $-2.80 \times 10^{3}$ \\
\hline 0.040 & $-3.04 \times 10^{3}(7.6)$ & $-5.64 \times 10^{3}(211.9)$ & $-4.61 \times 10^{3}(130.9)$ & $-2.95 \times 10^{3}(0.2)$ & $-2.94 \times 10^{3}(-0.4)$ & $-2.95 \times 10^{3}$ \\
\hline
\end{tabular}


TABLE IX. Frequency-dependent NR hyperpolarizabilities of $\gamma_{z z z z}^{\mathrm{nr}}(-2 \omega ; \omega, \omega, 0), \gamma_{z z z z}^{\mathrm{nr}}(-3 \omega ; \omega ; \omega, \omega)$, and $\gamma_{z z z z}^{\mathrm{nr}}(-\omega ; \omega,-\omega, \omega)$ of $p$-methylpyridone calculated at the HF/6-31G level using BK perturbation theory and various sets of harmonic TD-FICs. The exact result is included for sake of comparison. The percentage error with respect to the sum of the electronic and exact dynamic NR values are given in parentheses. Those cases where the error exceeds $5 \%$ are blocked off with dashed lines. All quantities are in a.u.

\begin{tabular}{|c|c|c|c|c|c|c|}
\hline$\omega$ & $(0 / 0)$ & $(\omega / \varnothing)$ & $(\omega / \omega)$ & $(\omega, 0 / \omega, 0)$ & $(2 \omega, \omega, 0 / 2 \omega, \omega, 0)$ & Exact \\
\hline \multicolumn{7}{|c|}{$\gamma_{z z z z}^{\mathrm{nr}}(-2 \omega ; \omega, \omega, 0)$} \\
\hline 0.005 & $-1.37 \times 10^{3}(-103.4)$ & $-9.41 \times 10^{3}(-14.3)$ & $1.27 \times 10^{4}(-259.4)$ & $1.38 \times 10^{4}(-271.2)$ & $-7.75 \times 10^{3}(-32.7)$ & $-1.07 \times 10$ \\
\hline 0.020 & $-3.31 \times 10^{3}(7.6)$ & $-7.57 \times 10^{2}(-160.2)$ & $-2.62 \times 10^{3}(-38.0)$ & $-3.19 \times 10^{3}(-0.1)$ & $-3.20 \times 10^{3}(0.0)$ & $-3.20 \times 10$ \\
\hline 0.040 & $-3.31 \times 10^{3}(5.6)$ & $-2.60 \times 10^{3}(-40.2)$ & $-2.93 \times 10^{3}(-18.7)$ & $-3.23 \times 10^{3}(0.2)$ & $-3.23 \times 10^{3}(0.0)$ & $-3.22 \times 10$ \\
\hline
\end{tabular}

$\gamma_{z z z Z}^{\mathrm{nr}}(-3 \omega ; \omega, \omega, \omega)$

\begin{tabular}{|c|c|c|c|c|c|c|}
\hline 0.005 & $7.62 \times 10^{3}(-175.8)$ & $-7.46 \times 10^{3}(-53.2)$ & $-8.74 \times 10^{3}(-42.8)$ & $-1.09 \times 10^{4}(-25.4)$ & $-1.10 \times 10^{4}(-24.5)$ & $-1.40 \times 10^{4}$ \\
\hline 0.020 & $2.17 \times 10^{2}(-38.0)$ & $1.65 \times 10^{3}(9.0)$ & $1.39 \times 10^{3}(0.5)$ & $1.38 \times 10^{3}(0.2)$ & $1.38 \times 10^{3}(0.0)$ & $1.38 \times 10^{3}$ \\
\hline 040 & $5.29 \times 10^{1}(-12.9)$ & $4.13 \times 10^{2}(5.2)$ & $3.11 \times 10^{2}(0.1)$ & $3.10 \times 10^{2}(0.0)$ & $3.10 \times 10^{2}(0.0)$ & $3.10 \times 10^{2}$ \\
\hline
\end{tabular}

$\gamma_{z z z z}^{\mathrm{nr}}(-\omega ; \omega,-\omega, \omega)$

\begin{tabular}{l|ccc|ccc}
0.005 & $2.44 \times 10^{4}(50.4)$ & $-1.82 \times 10^{4}(-195.4)$ & $-1.29 \times 10^{4}(-164.6)$ & $5.09 \times 10^{3}(-61.0)$ & $\left.1.34 \times 10^{2}(-89.6)\right]$ & $1.57 \times 10^{4}$ \\
0.020 & $6.09 \times 10^{3}(-18.4)$ & $2.20 \times 10^{3}(-59.3)$ & $3.95 \times 10^{3}(-40.9)$ & $7.84 \times 10^{3}(-0.1)$ & $7.84 \times 10^{3}(0.0)$ & $7.84 \times 10^{3}$ \\
0.040 & $5.86 \times 10^{3}(-4.6)$ & $7.35 \times 10^{2}(-69.5)$ & $2.82 \times 10^{3}(-43.1)$ & $6.22 \times 10^{3}(0.0)$ & $6.22 \times 10^{3}(0.0)$ & $6.22 \times 10^{3}$ \\
\hline
\end{tabular}

were evaluated for the most common cases using static FICs and the infinite optical frequency approximation. Tests of that approximation for finite frequencies are carried out here at the HF/6-31G level for three medium size conjugated organic molecules that are either nonpolar, or polar with a covalent ground state, or polar with a charge transfer ground state. At frequencies smaller than 0.06 a.u. $\left(\sim 13100 \mathrm{~cm}^{-1}\right.$ or $1.63 \mathrm{eV}$ ) errors in the infinite frequency approximation may become significant for the longitudinal (hyper)polarizabilities.

A possible alternative to the infinite-frequency approximation is to combine static FICs with the BK dynamic perturbation formulas. Although this procedure leads to some overall improvement, the extent of that improvement-if any-depends upon the molecule and the property. On the contrary, when appropriately chosen FD-FICs are combined with perturbation theory the result is exact at all frequencies. Furthermore, for the $\pi$-conjugated organic molecules studied in this paper the reduced set consisting of $\chi_{1,|0|}^{z}, \chi_{1,|\omega|}^{z}$ plus the harmonic $\chi_{2,|0|}^{z z}$ and $\chi_{2,|\omega|}^{z z}$ yields accurate values outside the IR region. This reduced set is complete for $\beta_{z z z}^{\mathrm{nr}}(-\omega ; \omega, 0)$ and $\gamma_{z z z z}^{\mathrm{nr}}(-\omega ; \omega, 0,0)$. The harmonic approximation is exact for first hyperpolarizabilities and yields an insignificant error for $\gamma_{z z z z}^{\mathrm{nr}}(-\omega ; \omega, 0,0)$ even within the IR region.

Of course, more molecules need to be examined before our conclusions based on the computations done here can be considered general. In addition, the effect of the basis set and the electron correlation must be investigated. We also plan to study other properties of interest such as sum-frequency generation.

\section{ACKNOWLEDGMENT}

Support for this work under Grant No. PB98-0457C02-01 from the Dirección General de Enseñanza Superior e Investigación Científica y Técnica (MEC-Spain) is acknowledged.
${ }^{1}$ B. Champagne and B. Kirtman, in Handbook of Advanced Electronic and Photonic Materials, edited by H. S. Nalwa (Academic, San Diego, 2001).

${ }^{2}$ D. M. Bishop and P. Norman, in Handbook of Advanced Electronic and Photonic Materials, edited by H. S. Nalwa (Academic, San Diego, 2001).

${ }^{3}$ M. C. Magnoni, P. Mondini, M. Del Zoppo, C. Castiglioni, and G. Zerbi, J. Chem. Soc., Perkin Trans. 2 2, 1765 (1999).

${ }^{4}$ P. Macak, Y. Luo, P. Norman, and H. Agren, J. Chem. Phys. 113, 7055 (2000).

${ }^{5}$ B. Kirtman and D. M. Bishop, Chem. Phys. Lett. 175, 601 (1990).

${ }^{6}$ D. M. Bishop and B. Kirtman, J. Chem. Phys. 95, 2646 (1991).

${ }^{7}$ D. M. Bishop and B. Kirtman, J. Chem. Phys. 97, 5255 (1992).

${ }^{8}$ D. M. Bishop, B. Kirtman, and B. Champagne, J. Chem. Phys. 107, 5780 (1997).

${ }^{9}$ D. M. Bishop, J. M. Luis, and B. Kirtman, J. Chem. Phys. 108, 10013 (1998).

${ }^{10}$ J. M. Luis, J. Martí, M. Duran, J. L. Andrés, and B. Kirtman, J. Chem. Phys. 108, 4123 (1998).

${ }^{11}$ J. M. Luis, M. Duran, and J. L. Andrés, J. Chem. Phys. 107, 1501 (1997).

${ }^{12}$ D. M. Bishop, M. Hasan, and B. Kirtman, J. Chem. Phys. 103, 4157 (1995).

${ }^{13}$ J. Martí and D. M. Bishop, J. Chem. Phys. 99, 3860 (1993).

${ }^{14}$ J. M. Luis, M. Duran, J. L. Andrés, B. Champagne, and B. Kirtman, J. Chem. Phys. 111, 875 (1999).

${ }^{15}$ B. Champagne, J. M. Luis, M. Duran, J. L. Andrés, and B. Kirtman, J. Chem. Phys. 112, 1011 (2000).

${ }^{16}$ E. Cammi, B. Mennucci, and J. Tomasi, J. Am. Chem. Soc. 120, 8834 (1998).

${ }^{17}$ P. Otto, A. Martinez, and J. Ladik, J. Chem. Phys. 111, 6100 (1999).

${ }^{18}$ B. Kirtman, J. M. Luis, and D. M. Bishop, J. Chem. Phys. 108, 10008 (1998).

${ }^{19}$ B. Kirtman, B. Champagne, and J. M. Luis, J. Comput. Chem. 21, 1572 (2000).

${ }^{20}$ D. M. Bishop and E. K. Dalskov, J. Chem. Phys. 104, 1004 (1996).

${ }^{21}$ O. Quinet and B. Champagne, J. Chem. Phys. 109, 10594 (1998).

${ }^{22}$ D. M. Bishop, and B. Kirtman, J. Chem. Phys. 109, 9674 (1998).

${ }^{23}$ J. M. Luis, M. Duran, B. Champagne, and B. Kirtman, J. Chem. Phys. 113, 5203 (2000).

${ }^{24}$ A sign error in Eqs. (12), (13), (18), (19), (22), (23), and (25) in Ref. 23 has been corrected.

${ }^{25}$ J. M. Luis, B. Champagne, and B. Kirtman, Int. J. Quantum Chem. 80, 471 (2000).

${ }^{26}$ B. Kirtman, B. Champagne, and J. M. Luis, J. Comput. Chem. 21, 1572 
(2000).

${ }^{27}$ P. Fischer, D. S. Wiersma, R. Righini, B. Champagne, and A. D. Buckingham, Phys. Rev. Lett. 85, 4253 (2000).

${ }^{28}$ B. Champagne, P. Fischer, and A. D. Buckingham, Chem. Phys. Lett. 331, 83 (2000).
${ }^{29}$ O. Quinet and B. Champagne, Int. J. Quantum. Chem. (to be published).

${ }^{30}$ D. M. Bishop, B. Champagne, and B. Kirtman, J. Chem. Phys. 109, 9987 (1998).

31 M. J. Frisch, G. W. Trucks, H. B. Schlegel et al., GAUSSIAN 98, Revision A.6 (Gaussian, Inc., Pittsburgh, 1998). 